\title{
Neonatal tetanus surveillance in Bayelsa state of Nigeria: a five-year review
}

\section{Abisoye S. Oyeyemi, Hilda C. Afakwu, Esievoadje Akpofure, Luke E. Izibekien}

Community Medicine, Niger Delta University, Yenagoa, Bayelsa, Nigeria

Objective

To assess the performance of neonatal tetanus surveillance in Bayelsa state of Nigeria.

Introduction

Neonatal tetanus (NT) though a preventable disease, remains a disturbing cause of neonatal morbidity and mortality particularly in low income countries where maternal and child care are substandard and antitetanus immunization coverage is still poor. The disease, which is mostly fatal, is particularly common in hard to reach and rural areas where deliveries take place at home or with untrained attendants without adequate sterile procedures and in unclean environment. Since eliminating NT became a global target, significant reductions in NT deaths have been reported. The most recent estimates by WHO (2015) put death of newborns due to NT at 34,019, a 96\% reduction from the situation in the late 1980 s.

All countries are committed to "elimination" of maternal and neonatal tetanus (MNT), i.e., a reduction of NT incidence to below one case per 1000 live births per year in every district. A strong neonatal tetanus surveillance (NTS) is however required to achieve this. As of March 2018, only 14 countries were yet to eliminate MNT and this includes Nigeria.

The different types of NTS recommended are conducted to varying degrees of efficiency and effectiveness in Nigeria under the major surveillance strategy - the Integrated Disease Surveillance and Response (IDSR). These include routine monthly surveillance, zero reporting, active surveillance and retrospective record review.

Nigeria comprises six geopolitical zones, 36 states and a Federal Capital territory (FCT), and is made up of 774 Local Government areas (LGA) (districts) - an LGA being the lowest administrative level. This study was conducted in Bayelsa state - one of the six states in the south zone. It is made up of eight LGAs, more than half of which are riverine and consists of many hard-to-reach communities, where formal functional health facilities are few and far between. Health workers are in short supply and funding of health care delivery is poor in the state.

\section{Methods}

This was a retrospective review of all confirmed cases of neonatal tetanus that were managed at the two tertiary hospitals in the state - Niger Delta University Teaching Hospital Okolobiri (NDUTH), and Federal Medical Centre Yenagoa (FMC) - between January 2009 and December 2013. These were the only two public facilities that had the capacity to manage NT cases in the state. Relevant data including sociodemographics, pregnancy and birth history of patients, cord care and tetanus toxoid immunization of mothers were abstracted from the case files. The cases were traced to the office of the State Epidemiologist, where all cases were expected to be documented and investigated in line with the existing neonatal tetanus surveillance. Ethical approval was obtained from the Research and Ethics Committee of NDUTH for the research and permission was given to access case files.

\section{Results}

A total of 48 cases were managed in both facilities (36/75.0\% in NDUTH and 12/25.0\% in FMC) in the period under review but only 13 cases $(27.1 \%)$ were reported to the office of the State Epidemiologist. Figure 1 shows the number of cases per year of review. The cases were resident in seven out of the eight LGAs. The mean age of cases was $8.98(\mathrm{SD}=5.14)$ days and $29(60.4 \%)$ were male while $19(39.6 \%)$ were female. Available evidence showed that only $2.1 \%$ of the cases were protected at birth (mothers had TT2+); $91.7 \%$ of mothers did not have antenatal care and all the mothers were delivered by traditional birth attendants; $70.8 \%$ had their umbilical cord cut with new (?sterile) blade; and $43.8 \%$ had their cord treated with methylated spirit, others were treated with just water or some herbal preparation. Educational attainment of mothers of cases was primary (54.2\%) and secondary (45.8\%).

SDS Annual Conference Proceedings 2019. This is an Open Access article distributed under the terms of the Creative Commons AttributionNoncommercial 4.0 Unported License (http://creativecommons.org/licenses/by-nc/3.0/), permitting all non-commercial use, distribution, and reproduction in any medium, provided the original work is properly cited. 


\section{Conclusions}

There were gaps in Neonatal Tetanus Surveillance in Bayelsa State as only $27.1 \%$ of cases were captured at the state level. Many mothers and their newborns were still not protected against tetanus, and delivery and cord care were done in unhygienic conditions. There is an urgent need to strengthen NT surveillance, improve vaccination against tetanus, and encourage skilled birth attendance in the state.

\section{Acknowledgement}

We thank the staff of the hospitals and that of the office of the State Epidemiologist who facilitated data collection.

\section{References}

1. WHO. Immunization, Vaccines and Biologicals: Tetanus. http://www.who.int/immunization/diseases/tetanus/en/. Accessed on $23 \mathrm{Jul} 2018$

2. WHO. Immunization, Vaccines and Biologicals: Maternal and Neonatal Tetanus Elimination (MNTE): The initiative and challenges.http://www.who.int/immunization/diseases/MNTE_initiative/en/ Accessed on $23 \mathrm{Jul}$ 2018.

3. WHO. WHO-recommended standards for surveillance of selected vaccine-preventable disease. WHO. 2003

4. Bayelsa State Ministry of Health. Health facilities and their distribution across the Local Government Areas of Bayelsa State. 2010.

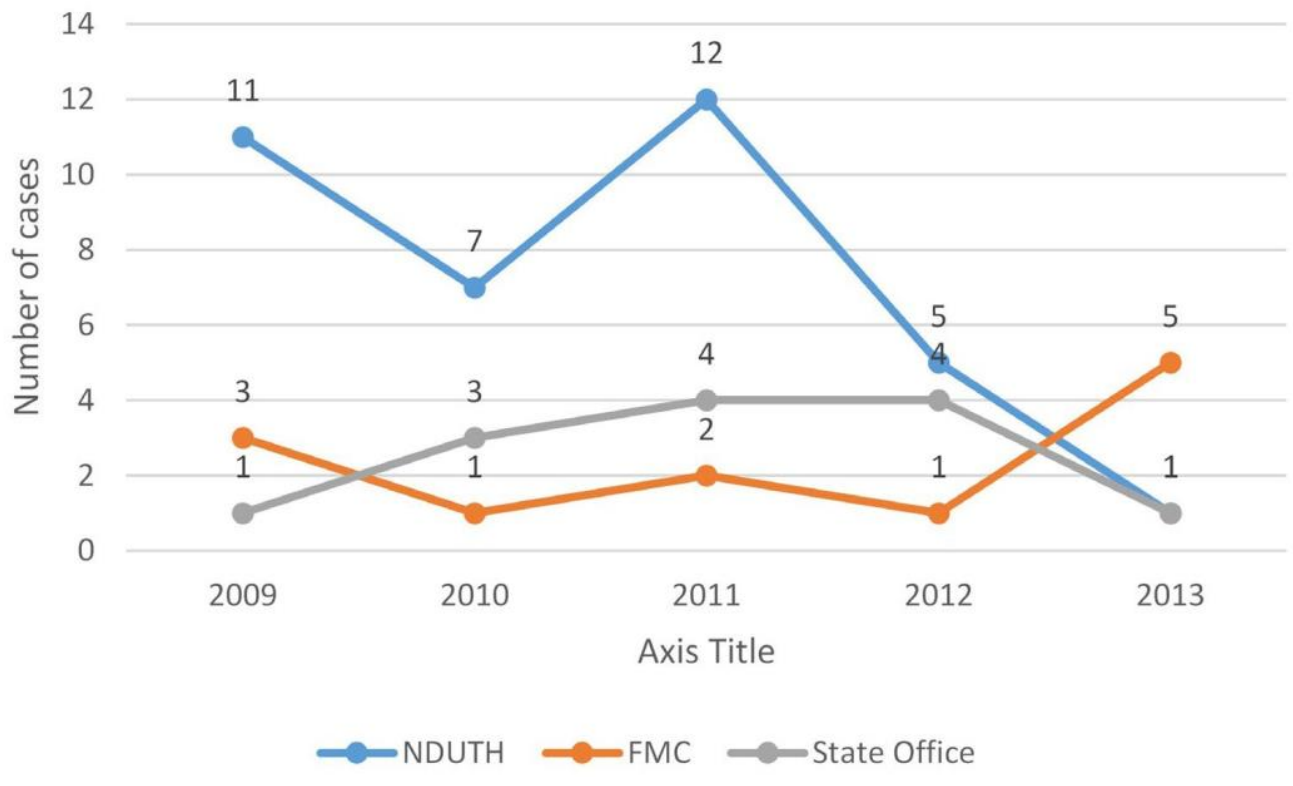

Figure 1. Number of cases seen at the facilities and reported to the office of the State Epidemiologist 